\title{
Grey-Zone Turbulence in the Neutral Atmospheric Boundary Layer
}

\section{Rachel Honnert ${ }^{1}$ (1D)}

Received: 6 February 2018 / Accepted: 28 September 2018 / Published online: 15 October 2018

(c) The Author(s) 2018

\begin{abstract}
The turbulence generated by wind shear is described at grey-zone resolutions using a theoretical neutral boundary layer based on atmospheric conditions constructed from measurements from the CASES-99 field campaign. Six-metre-resolution large-eddy simulations (LES) are performed to access the "true" resolved turbulence for two cases, corresponding to a forcing of the boundary layer by zonal geostrophic wind speeds of $10 \mathrm{~m} \mathrm{~s}^{-1}$ and $20 \mathrm{~m} \mathrm{~s}^{-1}$. The LES fields are subject to a coarse-graining procedure in order to compute turbulence diagnostics in the grey zone, with the robustness and weakness of various averaging procedures tested, for which simple top-hat averaging is found to be both suitable and accurate. In addition, the "true" resolved and subgrid-scale fluxes, variances, turbulent kinetic energy and production terms are quantified on various scales. The grey zone of turbulence is defined as the range of scales where 10-90\% of turbulence is resolved, which here ranges from resolutions of $25-800 \mathrm{~m}(0.03<\Delta x / h<1$, where $\Delta x$ is the horizontal resolution, and $h$ is the boundarylayer height). The subgrid/resolved partitioning of the variances of the velocity components depends on the geostrophic wind speed, which is not the case for the momentum-flux partitioning. Dynamic production terms show that fine-scale turbulence is isotropic $(\Delta x / h<0.03)$ and is quasi one-directional, oriented in the direction of the geostrophic wind vector at the mesoscale $(\Delta x / h>1)$. The turbulence parametrizations, which are tested in the Méso-NH model by running simulations at resolutions from the LES scale to the mesoscale, fail to produce the correct turbulence regardless of resolution.
\end{abstract}

Keywords Grey-zone turbulence $\cdot$ Large-eddy simulation · Neutral boundary layer

\section{Introduction}

The grey zone of turbulence is defined by Wyngaard (2004) as the range of scales where the resolution of the model $(\Delta x)$ is close to the size of the turbulent structures. At these resolutions, the turbulent boundary-layer structures are partly resolved and partly subgrid scale. Beare (2014) estimated the dominant length scales in the atmospheric boundary

Rachel Honnert

rachel.honnert@meteo.fr

1 CNRM UMR 3589, Météo-France/CNRS, 42, Av. Gaspard Coriolis,

31057 Toulouse Cedex 01, France 
layer (ABL) from large-eddy simulations (LES), and defined the grey zone of turbulence as the scale where both the inversion depth and the dissipation length scale are of similar magnitude. Honnert et al. (2011) studied the convective boundary layer (CBL) from LES results, and estimated that the grey zone may extend from $0.2 h$ to $2 h$, where $h$ is the boundary-layer height plus the depth of the cloud layer above, i.e the hectometric scales.

Honnert et al. (2011) presented theoretical similarity functions describing the resolved and subgrid-scale partitioning of turbulence, comparing these functions to actual simulations with a large set of parametrizations, to demonstrate that, in the free CBL, no parametrization is able to reproduce correctly the decrease in subgrid-scale turbulence existing at finer resolutions. Therefore, parametrizations must be adapted to the grey zone of turbulence.

Since then, while modifications of parametrizations have been made to run models at resolutions within the grey zone of turbulence, most of these modifications only concern the CBL. Boutle et al. (2014) blended a tri-dimensional Smagorinsky scheme with a unidimensional non-local ABL scheme with the help of the similarity functions proposed by Honnert et al. (2011), which were introduced to describe the CBL. Ito et al. (2015) extended the Mellor and Yamada (1982) parametrization by modifying the length scales using statistics obtained from a coarse-graining procedure LES investigations of the dry convective mixed layer in the free convective region. Shin and Hong (2015) quantified the local and non-local turbulence in the free CBL, and investigated the CBL forced by flow at scales in the grey zone in order to adjust the vertical profiles produced from their non-local K-gradient scheme. Dorrestijn et al. (2013) proposed a stochastic scheme for the grey zone of turbulence based on data of an oceanic CBL case.

Non-local thermal turbulence structures, which are the dominant and largest structures in the CBL, are the first structures to be partly resolved when model resolution increases. Thus, the parametrization of thermal turbulence is the first parametrization modellers have to deal with at grey-zone resolutions. Honnert and Masson (2014) proved, however, that dynamic turbulence is not negligible in the free or forced CBL at grey-zone resolutions.

Until now, the grey zone of turbulence of dynamic origin has not been investigated. The dynamic production of turbulence is of a different nature from the thermal production: turbulent coherent structures produced by free convection are large and vertical (CBL thermals), whilst the structures produced by wind shear are tilted and three-dimensional. These differences may affect both the range of scales concerning the grey zone and the parametrizations of the turbulence at these particular scales. Therefore, as shear-driven turbulence has to be studied at sub-kilometre scales, it is difficult, if not impossible, to separate dynamic and thermal turbulence in LES investigations based on real cases. Observations of purely dynamic turbulence are rare and perhaps unrealistic, which explains the necessity of using an idealized LES approach of neutral cases for the investigation of dynamic turbulence in the grey zone.

Investigated here is the grey zone in a neutral atmospheric boundary layer by means of a LES approach. In Sect. 2, the benchmark cases from the CASES-99 field campaign are presented. In Sect. 3, the subgrid-scale velocity variances, turbulent kinetic energy (TKE), and momentum fluxes are diagnosed in the grey zone of turbulence of a neutral case, as well as the dynamic turbulence-production terms. Sub-kilometre simulations are performed and compared with the LES reference case in order to test parametrizations in Sect. 4. The last section is dedicated to discussions and conclusions. 


\section{Benchmark Simulations}

\subsection{Reference Experimental Data}

The simulations are based on the CASES-99 experiment, which took place from 1 to 31 October 1999 in Kansas, USA, on a flat and homogeneous site. While the experiment was originally designed to investigate the stable ABL, and the morning and evening transition periods (Poulos et al. 2002), measurements were also made in near-neutral conditions, which can be used to simulate purely dynamic turbulence. The experimental data chosen here are described in Drobinski et al. (2007).

The prescribed roughness length is $0.1 \mathrm{~m}$, which generates a friction velocity of about $0.42 \mathrm{~m} \mathrm{~s}^{-1}$ at the prescribed wind speed. A constant $(293.15 \mathrm{~K})$ potential temperature is imposed up to an altitude of $750 \mathrm{~m}$ (Drobinski et al. 2007), and then a constant adiabatic gradient is applied up to $1500 \mathrm{~m}$ in height. A stable layer suppresses turbulence above an altitude of $750 \mathrm{~m}$ (initially) and limits the size of the eddies. The heat and humidity fluxes at the surface are zero during the simulations, and there is no water vapour in the simulation. The buoyancy flux is, therefore, zero inside the ABL during the simulations, and the production of turbulence is purely dynamic. The zonal geostrophic wind speed is prescribed for two sets of simulations of geostrophic wind speeds of $10 \mathrm{~m} \mathrm{~s}^{-1}$ and $20 \mathrm{~m} \mathrm{~s}^{-1}$, which are denoted as slow and fast simulations, respectively.

\subsection{Large-Eddy Simulations}

The Méso-NH model (Lafore et al. 1998) is used to simulate the neutral ABL cases on a $3.2 \times 3.2 \times 1.5 \mathrm{~km}^{3}$ cyclic domain over a flat and homogeneous terrain, with the upper $100 \mathrm{~m}$ of the domain absorbing gravity waves. Eddies remain smaller in size than $1000 \mathrm{~m}$ because they are contained within the ABL. The horizontal grid resolution is set to $6.25 \mathrm{~m}$ where, at this resolution, turbulence is mainly resolved, and the remaining subgrid turbulence is homogeneous and isotropic, while the vertical grid resolution is $6 \mathrm{~m}$. The grid boxes are almost cubic in form, which suits three-dimensional turbulence parametrizations using the Deardorff length scale (grid size limited by the magnitude of the stability). Cuxart et al. (2000) simulate very effectively the small three-dimensional eddies.

Figure 1a, b shows, respectively, the vertical profiles of the potential temperature, and the hodographs of the velocity vector of the two sets of simulations. Even if the temperature of the fast simulations slightly increases during the simulation, the profile remains neutral and quasi-constant in the ABL over the 5-h simulation period. However, the top of the ABL, however, varies and depends on the geostrophic wind speed. Figure $1 \mathrm{~b}$ shows the hodographs of the fast and slow simulations after $5 \mathrm{~h}$ of simulation time, presenting a regular rotation of the wind vector with height and an increase in wind speed typical of an Ekman layer. If the $\mathrm{ABL}$ height is defined as the lowest altitude where the hodograph arrows are parallel to the geostrophic wind direction, the ABL heights of the fast and slow simulations are $h \approx 1200 \mathrm{~m}$ and $h \approx 985 \mathrm{~m}$, respectively. 

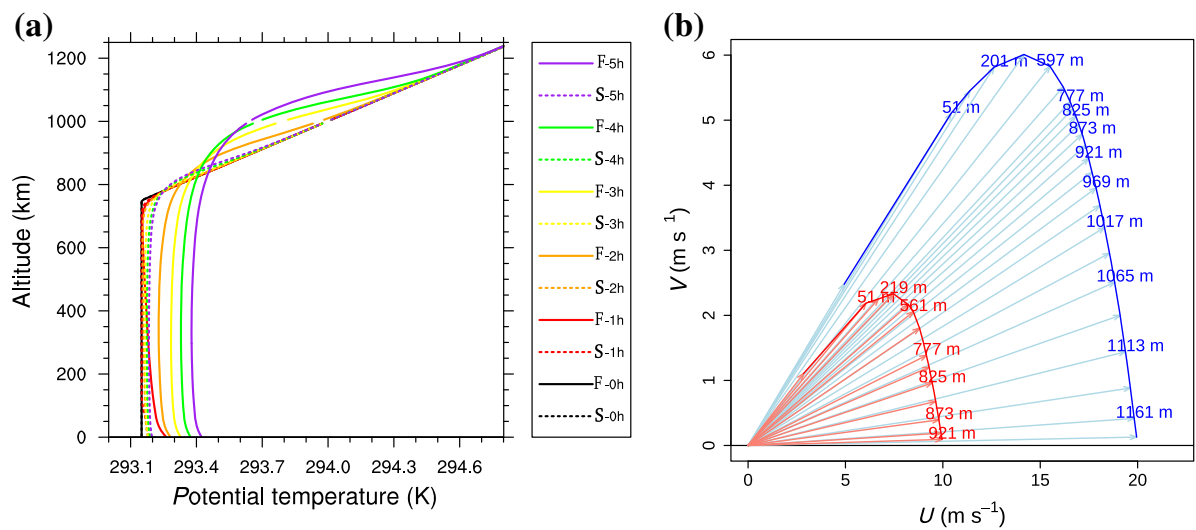

Fig. 1 a Vertical profiles of potential temperature during the slow ( $\mathrm{S}$, dashed lines) and fast (F, solid lines) simulations, and $\mathbf{b}$ hodographs ( $V$ : meridional velocity component in $\mathrm{m} \mathrm{s}^{-1}$ and $U$ : zonal velocity component in $\mathrm{m} \mathrm{s}^{-1}$ ) after $5 \mathrm{~h}$ of the slow (red) and fast (blue) simulations

\section{Results}

\subsection{Partial Similarity Functions}

Here, the "true" resolved and subgrid parts of turbulence as a function of the filtered scale are determined, where it is assumed, as in Honnert et al. (2011) for the CBL, that the ratio of the subgrid and total turbulence only depends on the value of $\Delta x / h$ according to

$$
\frac{e_{R E S}(\Delta x)}{e_{T O T}}=P_{R E S_{e}}\left(\frac{\Delta x}{h}\right),
$$

where $P_{R E S_{e}}$ is the partial similarity function of the resolved TKE $\left(e_{R E S}\right)$, which depends on $\Delta x$, where

$$
e_{R E S}(\Delta x)=0.5\left[{\overline{\left({\overline{u_{i}}}^{\Delta x}-<u_{i}>\right)^{2}}}^{\Delta x}\right] .
$$

Here, $u_{i}(i=1 \ldots 3)$ are the three velocity components, $<u_{i}>$ is the average of $u_{i}$ over the whole domain at a given altitude, and $\overline{u_{i}} \Delta x$ is the application of the top-hat filter on $u_{i}$ over an area defined by $\Delta x$. The Einstein notation is used in Eq. 2 and hereafter.

The "true" total TKE $e_{T O T}$ is computed as the sum of the resolved plus subgrid-scale TKE $\left(e_{R E S}(\mathrm{LES})\right.$ and $\left.e_{S G S}(\mathrm{LES})\right)$ of the large-eddy simulation and is independent of the model resolution according to

$$
e_{T O T}=e_{R E S}(\mathrm{LES})+e_{S G S}(\mathrm{LES})=e_{R E S}(\Delta x)+e_{S G S}(\Delta x),
$$

where the subgrid-scale TKE $e_{S G S}(\Delta x)$ is computed by subtracting $e_{R E S}(\Delta x)$ from $e_{T O T}$.

The ABL height is the altitude $h$ defined by

$$
e_{T O T}(h)=\frac{0.05}{h} \int_{0}^{h} e_{T O T}(z) \mathrm{d} z
$$

which is similar to the ABL height computed using the velocity profile in Sect. 2.2 (not shown). Figure 2 shows the subgrid-scale TKE $P_{S G S_{e}}=e_{S G S}(\Delta x) / e_{T O T}$, the subgrid-scale variances of the velocity components $\left(P_{S G S_{\frac{u^{12}}{}}}, P_{S G S} S_{v^{\prime 2}}\right.$ and $\left.P_{S G S_{w^{\prime 2}}}\right)$, and the subgrid-scale 

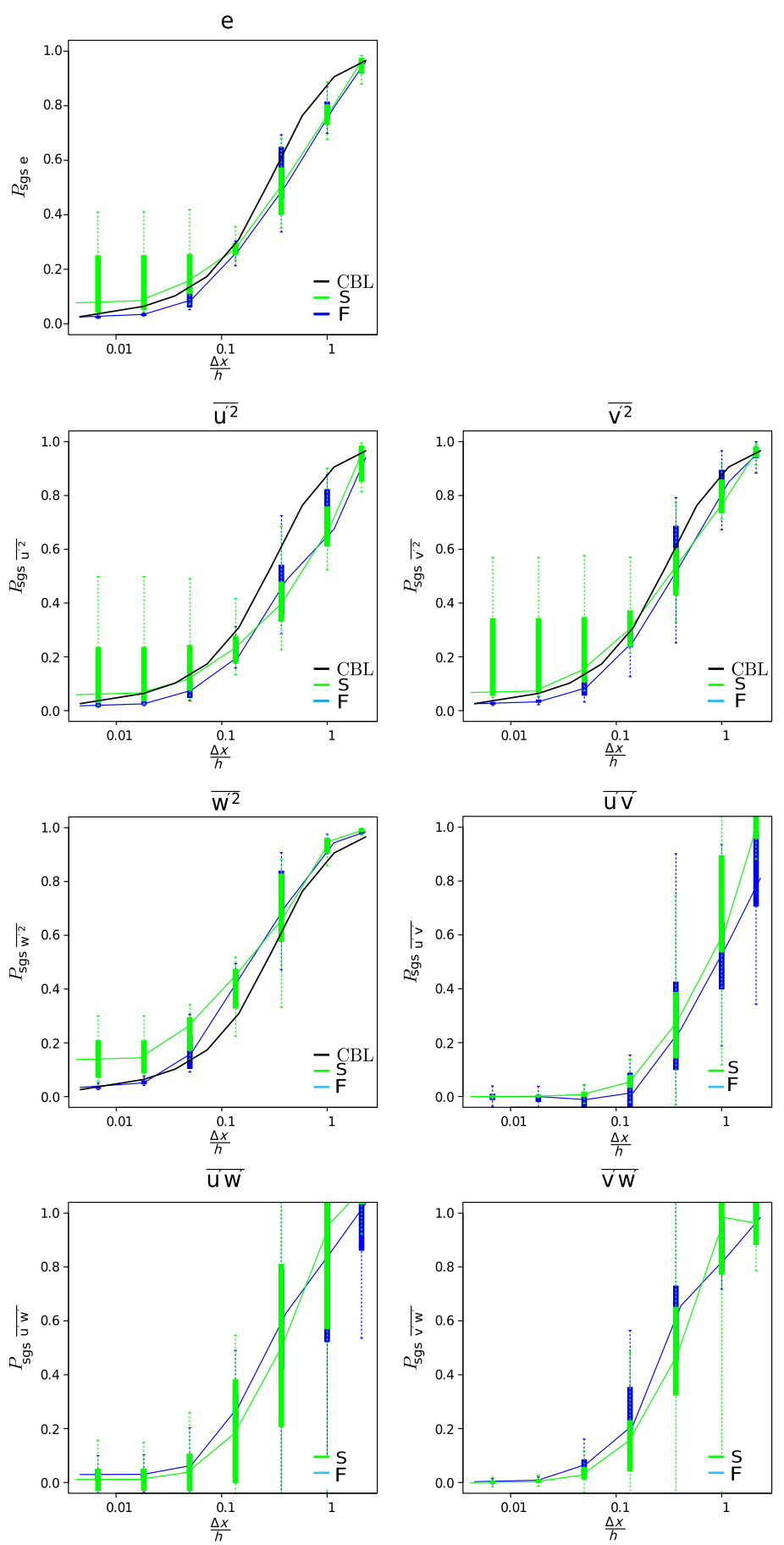

Fig. 2 Subgrid-scale TKE, variances of the velocity components, and momentum fluxes as a function of the grid size $(\Delta x)$ scaled by the ABL height $h$ calculated from the slow (green) and fast (blue) simulations. Partial similarity function for TKE from Honnert et al. (2011) (in black) 
dynamic fluxes $\left(P_{S G S_{\overline{u^{\prime} v^{\prime}}}}, P_{S G S_{\overline{u^{\prime} w^{\prime}}}}\right.$ and $P_{S G S_{\overline{v^{\prime} w^{\prime}}}}$ ) as a function of the scaled resolution $(\Delta x / h)$ for altitudes from 5 to $85 \%$ of the ABL height (the ABL height is greater in the fast simulation than in the slow). Boxplots present the dispersion per class of $\Delta x / h$ values for each windspeed forcing (fast simulation in light blue, and slow in green), while the curves link the medians of each class. In addition, the subgrid-scale TKE in the CBL (from Honnert et al. 2011) has been added.

The value of $P_{S G S_{e}}$ is small at fine resolutions, and close to one at the mesoscale. The grey zone of turbulence is defined here as that covering the range of scales for which the resolved turbulence is $10-90 \%$ of the total TKE. For the large scale $(\Delta x>800 \mathrm{~m}$, or $\Delta x / h>1)$, Fig. 2 shows that the total TKE is mainly composed of subgrid-scale TKE (resolved TKE less than 90\%). In this case, the TKE is mainly resolved (more than 90\%) for resolutions finer than $25 \mathrm{~m}$ (or $\Delta x / h<0.03$ ) in the slow and fast simulations. For the intermediate resolutions, the subgrid-scale turbulence increases with the grid resolution. The grey zone of turbulence of the neutral ABL can then be defined as the range of scales between $0.03<\Delta x / h<1$ (approximately $25-800 \mathrm{~m}$ ) in the slow and fast simulations. The resolved turbulence (not shown) equals the subgrid-scale turbulence at $\Delta x / h \approx 0.27$ (about $\Delta x \approx 250 \mathrm{~m}$ ). In the grey zone, the datasets present a large variability because the resolved TKE depends on the altitude, the size of the eddies, and the anisotropy, among other factors. Outside the grey zone, the variability is smaller.

To study the anisotropy of the turbulent motions in the ABL, the partial similarity functions of the variance of the velocity components are analyzed to give information on the size of the structures: at a given resolution, the larger the field structures, the more they are resolved. When the subgrid-scale turbulence is large, the structures are smaller than when the resolved turbulence is large. Figure 2 shows that the zonal velocity component (the free atmosphere) has larger structures than the meridional component, which has in turn larger structures than the vertical velocity component. The partitioning of the variances depends on the imposed geostrophic wind speed in the slow and fast simulations, with the latter structures rapidly resolved, while the subgrid-scale turbulence of the slow simulation is significant, even at high resolutions, which shows that the eddies are larger in the fast than in the slow simulation.

In contrast, the partitioning of the momentum fluxes (cf. Fig. 2) is the same for both the slow and fast simulations. At a given resolution, the intensity of the subgrid-scale exchange of momentum inside the ABL does not depend on the geostropic wind speed. The partitioning of the vertical fluxes $\left({\overline{u^{\prime} w^{\prime}}}^{\Delta x}\right.$ and ${\overline{v^{\prime} w^{\prime}}}^{\Delta x})$ is similar, while the subgrid-scale flux ${\overline{u^{\prime} v^{\prime}}}^{\Delta x}$ is smaller. Thus, the fields of $\overline{u^{\prime} w^{\prime}}$ and $\overline{v^{\prime} w^{\prime}}$ show structures of the same size; the $\overline{u^{\prime} v^{\prime}}$ field presents larger structures, as this flux is more resolved at a given resolution.

\subsection{Dynamic Production}

Honnert and Masson (2014) quantify the subgrid-scale production terms of turbulence in free and forced CBL cases when the turbulence is both thermal and dynamic. However, the thermal production is predominant even in cases when the CBL is forced by the wind speed; in the neutral ABL, the production is only dynamic. In this section, the production terms are computed in both simulations, with the subgrid-scale dynamic production terms at a given $\Delta x$ grid spacing 


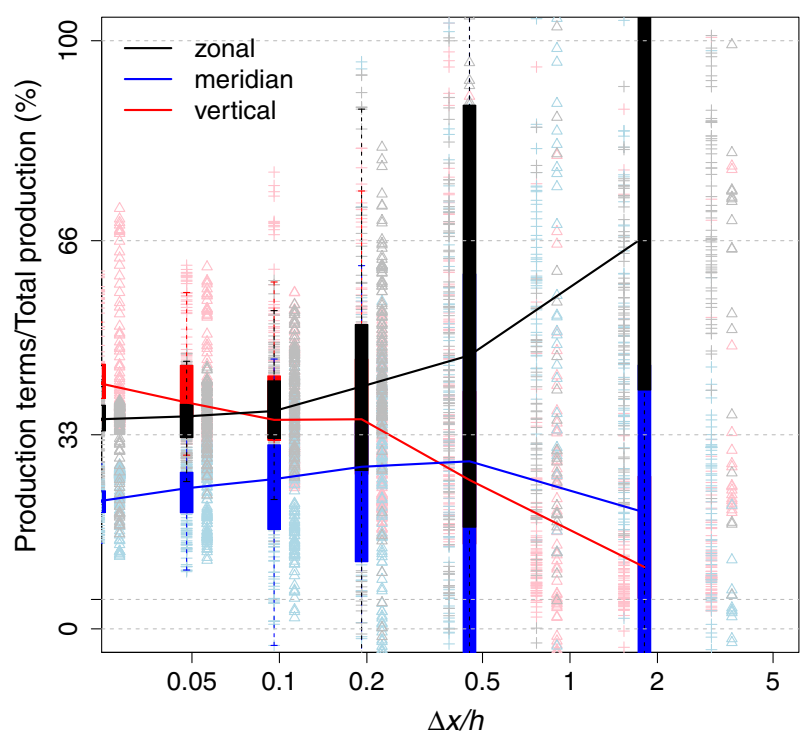

Fig. 3 Boxplot of percentage of zonal (black), meridional (blue) and vertical (red) dynamic production of turbulence per class of grid cell scaled by the ABL height after $5 \mathrm{~h}$ of simulation and at altitudes between 150 and $750 \mathrm{~m}$ for the slow and fast simulations. Data as a function of the grid cell scaled by the ABL height are in lighter colours for the slow (triangles) and fast (plus signs) simulations

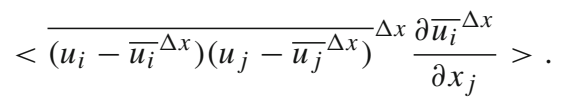

Hereafter, the zonal production is the sum of terms containing a gradient along $x$, the sum of the terms containing a gradient along $y$ forms the meridional production, whereas the vertical production is the sum of terms containing a vertical gradient.

Figure 3 shows the "true" percentages of the zonal, meridional and vertical dynamic production of turbulence as a function of the scaled resolution $(\Delta x / h)$. Boxplots present the dispersion of the production terms in each class of $\Delta x / h$. Although the boxplots summarize the production by the two LES in one unique group, the production terms are also shown by lighter colours in Fig. 3 (slow: triangles; fast: plus signs).

For fine resolutions $(\Delta x<25 \mathrm{~m}, \Delta x / h<0.03)$, the zonal, meridional and vertical production terms of turbulence are of the order of $33 \%$, implying that the turbulence is isotropic regardless of the geostrophic wind speed. For resolutions between 25 and $100 \mathrm{~m}$ $(0.03<\Delta x / h<0.1)$, the proportion of the vertical and meridional production of turbulence decreases, whilst the zonal production increases. For coarser resolutions $(\Delta x>100 \mathrm{~m})$, the zonal production is predominant, because it lies along the geostrophic wind direction. At a resolution of $3.2 \mathrm{~km}$ in the slow simulation, zonal production is responsible for about $70 \%$ of the total production. The turbulence is, therefore, isotropic for fine resolutions, while it is anisotropic for scaled resolutions coarser than 0.03 , with stronger dynamic production in the wind direction. 
(a)

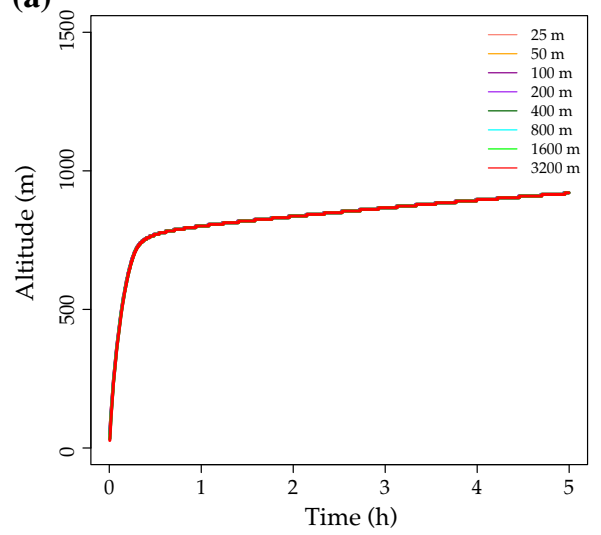

(b)

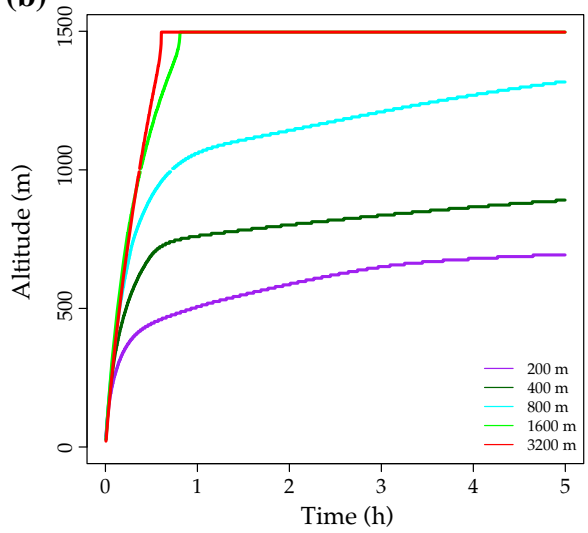

Fig. 4 The time evolution of ABL height for simulations at several resolutions (see the legend) with the a BL89 mixing length and a one-dimensional scheme, and the b Deardorff (DEAR) mixing length and a three-dimensional scheme. The geostrophic wind speed is $10 \mathrm{~m} \mathrm{~s}^{-1}$ as in the slow simulation

\section{Model Faults}

Consistent with Honnert et al. (2011), the LES and coarse-grained results are compared with simulations running at resolutions in the grey zone: simulations are performed for each wind speed $\left(10\right.$ and $\left.20 \mathrm{~m} \mathrm{~s}^{-1}\right)$, with nine resolutions $(12.5,25,50,100,200,400$, 800,1600 and $3200 \mathrm{~m}$ ), and two mixing lengths, namely those of Bougeault and Lacarrère (1989) (hereafter BL89) and of Deardorff (1972) and uni-directional (one-dimensional) vertical and three-dimensional isotropic turbulence schemes. The vertical resolution remains at $6 \mathrm{~m}$, as in the LES model. The BL89 mixing length is a mixing length that represents the size of the largest eddies at each altitude, and is usually used to simulate non-local turbulence in the CBL. The Deardorff mixing length uses the size of the grid cell $(\Delta x \Delta y \Delta z)^{1 / 3}$, where $\Delta x$ and $\Delta y$ are the two horizontal resolutions, and $\Delta z$ is the vertical resolution. The Méso-NH three-dimensional scheme is a complete set of second-order turbulence equations usually used in LES investigations with the Deardorff mixing length. The onedimensional scheme only computes the vertical equations, and is usually used with the BL89 mixing length at the mesoscale, since horizontal gradients are small in the K-gradient scheme.

The simulations are compared with the LES results and to the averaged LES fields, giving a similar performance regardless of the magnitude of the geostrophic wind speed. The ABL height is calculated according to Eq. 4. In the LES results, after $1 \mathrm{~h}$ of spin-up, the ABL height slightly increases (from 750 to $850 \mathrm{~m}$ altitude in the slow simulation, see Fig. 1a) during the 5-h simulation period, and this behaviour is perfectly reproduced by the BL89 mixing length (one- or three-dimensional) at all resolutions. The BL89 mixing length produces entirely subgrid-scale turbulence regardless of the resolution (see Fig. 5), which is inaccurate in the grey zone, and at the LES resolution, but which produces the correct ABL height (see Fig. 4a). In contrast, with the Deardorff mixing length in both the one- and three-dimensional turbulence schemes, the subgrid-scale turbulence and ABL height strongly depend on the model resolution (see Fig. 4b). Figure $4 \mathrm{~b}$ shows that the coarser the resolution, the greater the ABL height, where the value of $h$ is computed using Eq. 4 as the height at which the total TKE is $5 \%$ of the average total TKE below $h$. This diagnostic approach assumes that the total TKE 
DEAR-3D-12.5m

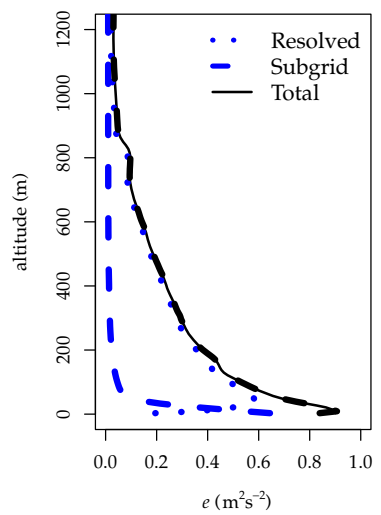

BL89-1D-12.5m

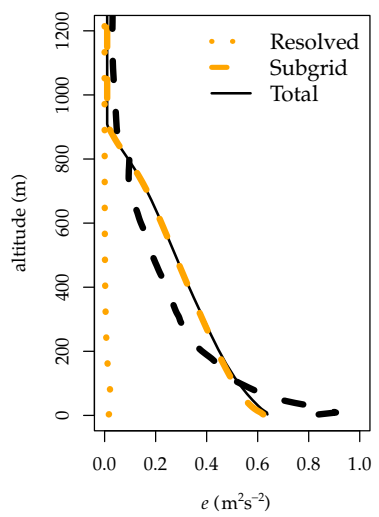

DEAR-3D-400m

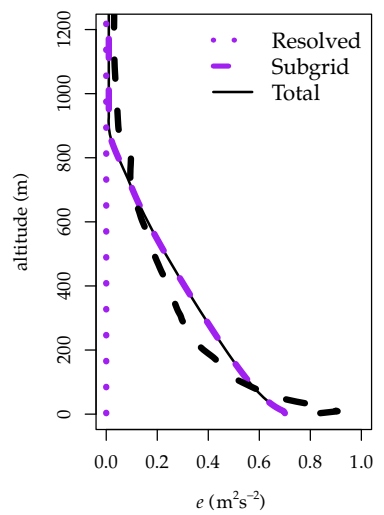

BL89-1D-400m

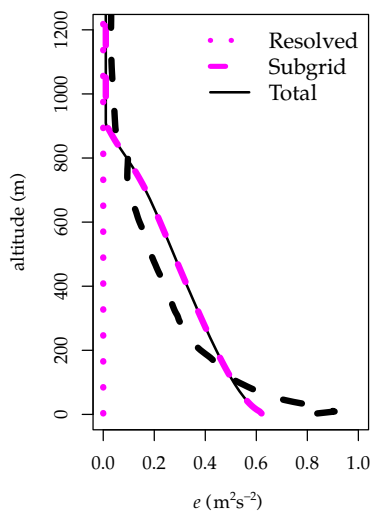

DEAR-3D-1600m

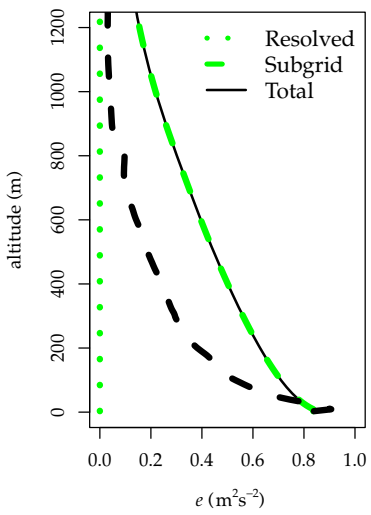

BL89-1D-1600m

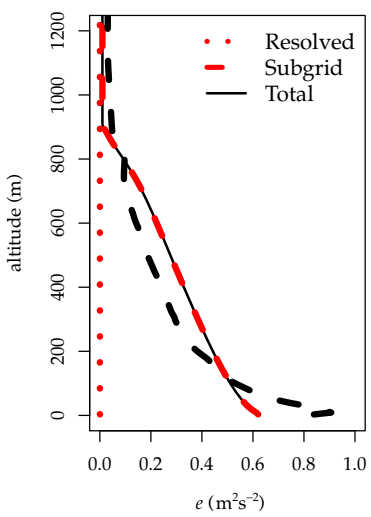

Fig. 5 Profiles of subgrid (dotted points), resolved (dashed lines) and total TKE, $e$ (in black) for the threedimensional scheme with the Deardorff mixing length (DEAR-3D) and the one-dimensional scheme with the BL89 mixing length (BL89-1D) at resolutions of 12.5, 400 and $1600 \mathrm{~m}$ resolution in the slow simulation. The total TKE of the LES results is indicated by a black dashed line

is large in the ABL and approximately null in the free atmosphere; however, the TKE is not zero in the free atmosphere (see Fig. 5). In the CBL, the lack of mixing is compensated by the resolved turbulence (see Honnert et al. 2011), but the total TKE in the neutral ABL is too large and too close to the total TKE in the free atmosphere at large scales. The limit between the turbulence in the ABL or in the free atmosphere is, therefore, unclear, as this diagnostic does not detect the value of $h$, but the top of the model, or overestimates the value of $h$. At a resolution finer than $400 \mathrm{~m}$, on the contrary, the Deardorff mixing length produces excessive turbulence, especially close to the surface, and as a consequence, the ABL height is too small compared with the LES results, and is not defined for resolutions smaller than $200 \mathrm{~m}$.

The conclusions are similar with reference to the rotation of the wind vector in the ABL (not shown). A rotation of the wind vector is shown in Fig. 1b, and is well-reproduced by the simulations with the BL89 mixing length regardless of the resolution. With the Deardorff 

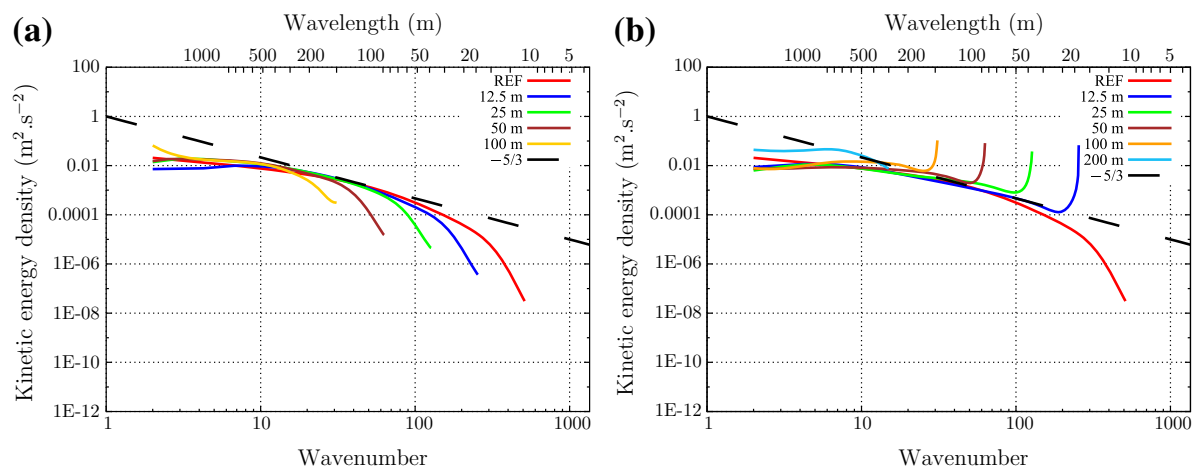

Fig. 6 The spectra of TKE in simulations at several resolutions (see the legend) with the Deardorff mixing length (DEAR) with the a three-dimensional (3D) or b one-dimensional scheme (1D)

mixing length, however, the coarser the resolution, the smaller the magnitude of the rotation, and, when the resolution is finer than $200 \mathrm{~m}$, the wind direction becomes chaotic.

The simulations with the Deardorff mixing length are considered as unsuitable for the large scales at resolutions coarser than $200 \mathrm{~m}$. However, despite the erroneous ABL height and wind-direction rotation, spectral analysis (Ricard et al. 2013) with the Deardorff mixing length and three-dimensional simulation in Fig. 6 exhibits results similar to the LES results for heights up to $100 \mathrm{~m}$. The ratio between the effective resolution (Ricard et al. 2013) and the model resolution appears to be constant, and is similar to the LES counterparts. The one-dimensional Deardorff simulation (DEAR-1D) presents an accumulation of energy at small scales while the BL89 simulations never produce the resolved fields necessary for a spectral analysis (not shown).

Furthermore, the simulations at several resolutions have been compared with the averaged fields of the LES results. Despite the good representation of the turbulence in the spectral space, and since the ABL height and the wind rotation are ill-represented at all resolutions with the Deardorff mixing length, this mixing length is considered as inapplicable in the grey zone. However, results are similar with the BL89 mixing length regardless of the averaging procedure (see Appendix 1), geostrophic wind speed, or resolution: the BL89 mixing length never produces resolved turbulence.

\section{Conclusion}

Grey-zone turbulence of dynamic origin is studied for two LES cases corresponding to a neutral ABL forced at the domain top by two different geostrophic wind speeds. The simulations are subject to a coarse-graining procedure (see Appendix 1). The "true" subgridscale/resolved partitioning of the TKE, variances of the velocity components, momentum fluxes, as well as the dynamic production terms, are computed, with results showing that, below a $25-\mathrm{m}$ resolution (about $\Delta x / h=0.03$ ), the turbulence is mainly resolved and the subgrid-scale turbulence is isotropic. In contrast, the turbulence is entirely found at the subgrid scale at resolutions coarser than 800 -m resolution (about $\Delta x / h=1$ ). There is a grey zone of turbulence in a neutral ABL, which extends from a $25-\mathrm{m}$ to a $800-\mathrm{m}$ resolution where the turbulence is partly resolved but non-isotropic. The size of the structures, and thus the definition of the grey zone, depends on the geostrophic wind speed: the greater the wind 
speed, the larger the structures. When the resolution increases, the larger structures are the first to be resolved and they enter the grey zone. At a given resolution, the magnitude of the subgrid-scale momentum fluxes inside the ABL does not depend on the geostrophic wind speed. The horizontal flux is resolved at coarser scales than the vertical momentum fluxes.

In order to test current parametrizations in the grey zone for horizontal dynamic turbulence, simulations are systematically made for nine horizontal resolutions from $12.5 \mathrm{~m}$ to $3.2 \mathrm{~km}$ with the BL89 and Deardorff mixing lengths and with one- and three-dimensional schemes, with results showing that the neutral ABL is ill-represented, especially at large scales. While this may not currently affect many numerical weather prediction models, as their resolutions are coarser than the grey zone of the neutral $\mathrm{ABL}$, the neutral $\mathrm{ABL}$ is an interesting theoretical state. In the grey zone and at a resolution of a few hundred metres, the subgrid-scale turbulence is (partly) dynamic even in the free CBL since ABL thermals are (partly) resolved. It has been proven, however, that, at those resolutions, the turbulence is not isotropic, and most of the turbulence schemes are incorrect. An adaptation of three-dimensional isotropic-turbulence schemes to the grey zone of turbulence is, therefore, necessary at hectometric scales.

Acknowledgements I would like to acknowledge Guillem Coquelet, Julien Leger and Clément Blot for their contributions, and Pascal Masquet for his careful comments.

Open Access This article is distributed under the terms of the Creative Commons Attribution 4.0 International License (http://creativecommons.org/licenses/by/4.0/), which permits unrestricted use, distribution, and reproduction in any medium, provided you give appropriate credit to the original author(s) and the source, provide a link to the Creative Commons license, and indicate if changes were made.

\section{Appendix 1}

\section{Averaging Procedure}

It is commonly assumed that the resolved fields simulated at high resolution can be considered as the "true" turbulence (Deardorff 1972), implying the intensity and size of ABL structures are statistically similar to those observed. This is not the case in the grey zone, where the resolved turbulence presents other characteristics not considered by the LES approach, whose resolution is strongly dependent on the chosen parametrization (Honnert et al. 2011, 2016). Here, the "true" ABL fields from the LES results are averaged to obtain "true" resolved fields at coarser resolutions, using the coarse-graining procedure of Shutts and Palmer (2006), Honnert et al. (2011) and Shin and Hong (2013). However, the averaging procedure differs between authors, with the difference mostly in the definition of the resolved field in terms of the cut-off-filter truncation in spectral space, whilst in the model, the grid spacing is set in real space. For these reasons, several averaging procedures are tested.

\section{Top-Hat Filter}

The top-hat filter performs nearest-neighbour filtering on a grid point with a rectangular function. It is easy to implement, and produces grid-like fields, such as those produced by models, which makes it easier to compare the LES average with model simulations in the grey zone. The real-space representation of a rectangular function, however, is the cardinal sine function, which has the drawback of incorporating non-local frequencies into the averaged field. 


\section{Moving Averaging}

The moving average is a top-hat filter shifting from point to point, which has the same inconvenience as the top-hat filter and, furthermore, the produced fields are fuzzy and not as comparable to the simulation as the top-hat method.

\section{Gaussian Filter}

Moeng et al. (2009) used a Gaussian filter to separate filter and subfilter scales, which is a moving-average procedure, where the weighted function is a Gaussian function. For a point $(x, y)$, the average of a parameter $c$ can be written as

$$
\bar{c}(x, y)=\iint c(x-\eta, y-\zeta) G(x-\eta, y-\zeta) \mathrm{d} \eta \mathrm{d} \zeta,
$$

where $G$ is the Gaussian function,

$$
G(x-\eta, y-\zeta)=\frac{6}{\Pi} \frac{1}{\left(\Delta_{f}\right)^{2}} \exp \left(\frac{-6\left[(x-\eta)^{2}+(y-\zeta)^{2}\right]}{\left(\Delta_{f}\right)^{2}}\right)
$$

where $\Delta_{f}$ is the size of the filter.

Moeng et al. (2009) explain that the Gaussian filter has the advantages of being applied in real space as the top-hat filter (cf. Sect. 5) without generating the noise near the cut-off scale by the sharp-wave cut-off filter. While the generated structures have more realistic shapes than those produced by the top-hat filter, the Gaussian filter smooths the fields more rapidly than the moving average, when the filter increases.

\section{Spectral Averaging}

The desired frequency band is selected in Fourier space by a top-hat filter where a lower and higher frequency are specified. Spectral averaging is easy to implement digitally.

\section{Discussion}

Figure 7 shows the four averaging procedures tested on the zonal velocity component at 200-m height, showing a similar behaviour in the grey zone. At fine scales, small structures are visible, but disappear as the grid size increases. All three velocity components display a different behaviour (not shown). The zonal velocity component has large structures, visible up to a resolution of $800 \mathrm{~m}$, while the meridional velocity component produces structures visible up to a resolution of 400, and the vertical velocity component up to 200-m resolution. This difference is due to the zonal geostrophic wind speed, which zonally stretches the ABL turbulence.

Although the top-hat filter does not conserve the shape of the ABL structures, and though the Gaussian filter seems to produce finer structures, the difference in intensity and size of the structures produced by the different averaging procedures is insignificant in this case. The impact on fluxes and turbulence diagnostics does not differ in our case. The results, therefore, produced by the top-hat filter have been selected for use. 

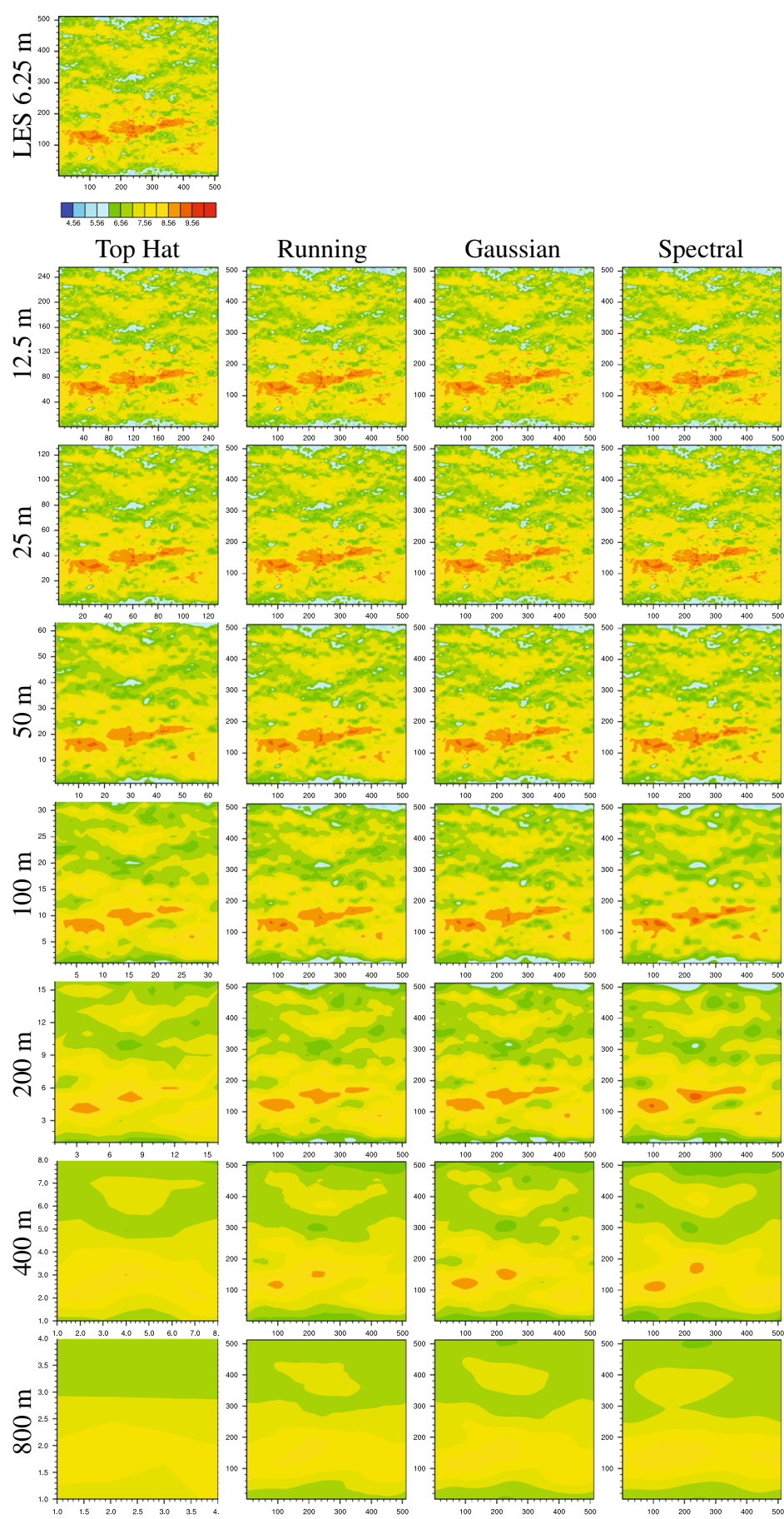

Fig. 7 Horizontal cross-sections of zonal wind speed in the slow simulation at a height of $200 \mathrm{~m}$ after $5 \mathrm{~h}$ of simulation, and the filtered fields by the top-hat, running, Gaussian and spectral methods at resolutions from 12.5 to $800 \mathrm{~m}$ 


\section{References}

Beare RJ (2014) A length scale defining partially-resolved boundary-layer turbulence simulations. BoundaryLayer Meteorol 151:39-55

Bougeault P, Lacarrère P (1989) Parametrisation of orography-induced turbulence in a mesobeta-scale model. Mon Weather Rev 117:1872-1890

Boutle IA, Eyre JEJ, Lock AP (2014) Seamless stratocumulus simulation across the turbulent grey zone. Mon Weather Rev 142:1655-1668

Cuxart C, Bougeault P, Redelsperger JL (2000) A turbulence scheme allowing for mesoscale and large-eddy simulations. Q J R Meteorol Soc 126:1-30

Deardorff JW (1972) Numerical investigation of neutral and unstable planetary boundary layers. J Atmos Sci 29:91-115

Dorrestijn J, Crommelin DT, Siebesma AP, Jonker HJ (2013) Stochastic convection parameterization estimated from high-resolution model data. Theor Comput Fluid Dyn 27:133-148

Drobinski P, Carlotti P, Redelsperger JL, Banta R, Masson V, Newsom R (2007) Numerical and experimental investigation of the neutral atmospheric surface layer. J Atmos Sci 64:137-156

Honnert R, Masson V (2014) What is the smallest physically acceptable scale for 1D turbulence schemes? Front Earth Sci 2:27. https://doi.org/10.3389/feart.2014.00027

Honnert R, Masson V, Couvreux F (2011) A diagnostic for evaluating the representation of turbulence in atmospheric models at the kilometric scale. J Atmos Sci 68:3112-3131

Honnert R, Couvreux F, Masson V, Lancz D (2016) Sampling of the structure of turbulence: implications for parameterizations at sub-kilometric scales. Boundary-Layer Meteorol 160:133-156. https://doi.org/10. 1007/s10546-016-0130-4

Ito J, Niino H, Nakanishi M, Moeng C-H (2015) An extension of Mellor-Yamada model to the terra incognita zone for dry convective mixed layers in the free convection regime. Boundary-Layer Meteorol 157:23-43

Lafore JP, Stein J, Ascencio N, Bougeault P, Ducrocq V, Duron J, Fischer C, Héreil P, Mascart P, Masson V, Pinty JP (1998) The Méso-NH atmospheric simulation system. Part I: adiabatic formulation and control simulation. Ann Geophys 16:90-109

Mellor GL, Yamada T (1982) Development of a turbulence closure model for geophysical fluid problems. Rev Geophys 20(4):851-875

Moeng C-H, LeMone MA, Khairoutdinov MF, Krueger SK, Bogenschutz PA, Randall DA (2009) The tropical marine boundary layer under a deep convection system: a large-eddy simulation study. J Adv Model Earth Syst. https://doi.org/10.3894/JAMES.2009.1.16

Poulos G, Blumen W, Fritts DC, Lundquist JK, Sun J, Burns SP, Nappo C, Banta R, Newsom R, Cuxart J, Terradellas E (2002) CASES-99: a comprehensive investigation of the stable nocturnal boundary layer. Bull Am Meteorol Soc 83:555-581

Ricard D, Lac C, Legrand R, Mary A, Riette S (2013) Kinetic energy spectra characteristics of two convectionpermitting limited-area models AROME and MesoNH. Q J R Meteorol Soc 139:1327-1341

Shin H, Hong S (2013) Analysis on resolved and parameterized vertical transports in the convective boundary layers at the gray-zone resolution. J Atmos Sci 70:3248-3261

Shin H, Hong S (2015) Representation of the subgrid-scale turbulent transport in convective boundary layers at gray-zone resolutions. Mon Weather Rev 143:250-271

Shutts GJ, Palmer TN (2006) Convective forcing fluctuations in a cloud-resolving model: relevance to the stochastic parameterization problem. J Clim 20:187-202

Wyngaard J (2004) Toward numerical modelling in the Terra Incognita. J Atmos Sci 61:1816-1826 